\title{
Millisecond Optical Phase Modulation Using Multipass Configurations with Liquid-Crystal Devices
}

\author{
Yihan Jin $\odot,{ }^{1}$ Steve J. Elston, ${ }^{1,}{ }^{*}$ Julian A.J. Fells $\odot,{ }^{1}$ Martin J. Booth, ${ }^{1}$ Chris Welch, ${ }^{2}$ Georg H. Mehlø, ${ }^{2}$ \\ and Stephen M. Morris ${ }^{1, \dagger}$ \\ ${ }^{1}$ Department of Engineering Science, University of Oxford, Parks Road, Oxford OX1 3PJ, United Kingdom \\ ${ }^{2}$ Department of Chemistry, University of Hull, Hull HU6 7RX, United Kingdom
}

(Received 16 April 2020; revised 17 June 2020; accepted 25 June 2020; published 5 August 2020)

\begin{abstract}
We present two configurations for analog 0 to $2 \pi$ optical phase modulation using liquid crystals (LCs), each of which achieve switching times that are $1 \mathrm{~ms}$ or less. One configuration is based on the switching behavior of a so-called nematic pi cell, and the other is based on the flexoelectro-optic effect in chiral nematic LCs when operated in the uniform lying helix geometry. Both configurations exploit a multipass optical arrangement to enhance the available optical phase range, while maintaining a fast switching speed. Moreover, these devices can be operated at or close to room temperature. Experimental data are found to be in good agreement with results predicted from theory for these multipass phase-modulation configurations.
\end{abstract}

DOI: 10.1103/PhysRevApplied.14.024007

\section{INTRODUCTION}

Optical phase modulation is important in many aspects of optical and optoelectronic technology [1]. Phase modulation can be obtained either by changing the refractive index (or effective refractive index) of a material through which light is propagating or by physically changing the optical path length of the light. For applications requiring a spatially structured phase modulation of a propagating optical wave front, a liquid crystal (LC) spatial light modulator (SLM) is often desirable and is employed in applications such as micromanipulation and beam shaping [2-7]. Commercial SLM technology based upon LCs broadly breaks down into two categories: those that provide digital, binary, or quantized phase modulation with a very limited number of states (e.g., 0 and $\pi$ phase states are commonly chosen) and those that provide analog (or at least, multilevel) phase modulation (e.g., a phase range of 0 to $2 \pi$ is commonly used). As an example, binary SLM technology [8-10] is often based on the bistable switching of ferroelectric LCs and can achieve switching times that are substantially less than $1 \mathrm{~ms}$. A high-speed technology based on antiferroelectric LCs has recently been demonstrated, but this uses rather thick layers of LCs, and thus, limits the potential pixel pitch [11]. Analog SLM technology, on the other hand, typically involves switching a layer of nematic LCs, but the switching times are generally much longer, of many milliseconds [12,13], although with

\footnotetext{
*steve.elston@eng.ox.ac.uk

†stephen.morris@eng.ox.ac.uk
}

careful material and device optimization $2 \mathrm{~ms}$ response times have been obtained in the laboratory [14].

Improving the switching speed, while maintaining access to a full range of phase states (from 0 to $2 \pi$ ), enables SLM technology to be used in a wider range of systems, such as in faster optical laser beam tracking and steering [15], and in higher-speed optical aberration correction in imaging systems [16]. There has been much work in this area, with a number of proposals and demonstrations of configurations that can enhance the switching speed (reduce the response time) in a useful way. For example, two-photon laser writing techniques have been employed to engineer advanced structures inside of LC devices to optimize switching times [17-19], and there has been work on incorporating polymer networks into LC layers to obtain submillisecond switching times [20].

An alternative approach has been demonstrated using the flexoelectro-optic effect in chiral nematic LCs [21]. This electro-optic effect, which can be observed when an electric field is applied perpendicular to the helical axis of the chiral nematic LC, can exhibit fast electro-optic properties because the characteristic length scale is the helical pitch (which can be of the order of 100s nm), rather than the device thickness. While the electro-optic effect offers submillisecond switching times, the optical response is generally quite small for most LC compounds and mixtures, with switching angles of just a few degrees. Bimesogenic LCs, however, can show much larger switching angles, allowing useful electro-optic effects to be exploited [22]. Using a configuration based upon the flexoelectrooptic effect, in combination with wave plates and a reflecting element, we have shown in a previous study that it is 
possible to achieve submillisecond 0 to $2 \pi$ optical phase modulation [23].

A drawback with the approach demonstrated in Ref. [23] is that the devices have to be operated at somewhat elevated temperatures (in excess of $100^{\circ} \mathrm{C}$ ), which is impractical for most technological applications. Unfortunately, room-temperature materials showing a large and high-speed flexoelectro-optic effect are not yet commonly available. A LC that shows substantial switching angles at room temperature has recently been demonstrated, but it requires large electric fields (equivalent to $100 \mathrm{~V}$ across a $5-\mu \mathrm{m}$-thick device to achieve a switching angle of $40^{\circ}$ ) [22]. Therefore, there is still considerable value in identifying new modes and configurations that can lead to fast switching and full $2 \pi$ phase modulation. The focus of this work, therefore, is to report on two alternative approaches that potentially address the shortcomings that are present in existing technologies, namely, full $2 \pi$ phase modulation operating at switching frequencies much greater than $100 \mathrm{~Hz}$ at room temperature.

\section{ALTERNATIVE CONFIGURATIONS}

In the first configuration, a nematic electro-optic mode is used in a high-speed regime. For nematic LCs, the "switchoff" speed is typically the limiting factor in engineering a high-speed optical response. This is because the "switch on" is driven by an applied voltage and the resulting switching speed is primarily controlled by the corresponding electric field and the viscosity of the LC. Therefore, by ensuring that the field is sufficiently large, high-speed switching can be obtained. However, the switch-off speed [24] is primarily controlled by the elasticity and viscosity of the LC, and therefore, this is harder to control, other than through material parameter optimization [25]. Alternatively, the switching speed can be improved through the use of polymer stabilization [26] or by making the device thinner. Polymer stabilization, while it can be effective, often leads to larger driving voltages and increased light scattering, both of which are unwanted side effects. Making the device thinner is a possible solution, as the thickness of a nematic LC layer strongly influences the switching speed. In general, the characteristic switch-off time constant, $\tau$, is given by $\tau \approx \gamma d^{2} / \pi^{2} K$ [24], where $\gamma$ is the rotational viscosity, $K$ is an elastic constant, and $d$ is the layer thickness. Therefore, a thin layer thickness $(d)$ leads to high-speed switching.

There are, however, two problems with the approach of reducing the layer thickness: (i) thin uniform layers are more difficult to engineer, and the devices become difficult to capillary fill; and (ii) thin layers show a reduced electrooptic effect, so the degree of phase modulation available may be insufficient. Here, we aim to address these two issues. First, we use a LC layer with a conventional device thickness of $5 \mu \mathrm{m}$, but exploit the switching of a thin region near the surfaces of the device. Second, we use a multipass optical arrangement to enhance the electro-optic effect at the surfaces of the device to obtain a full 0 to $2 \pi$ optical phase modulation.

For the second configuration, a room-temperature flexoelectro-optic device is employed that exhibits smallangle deflection, but the electro-optic response is enhanced (or amplified) through a multipass configuration. In our previous work using the flexoelectro-optic effect, we have shown that by integrating the LC layer between quarterwave plates and a mirror it is possible to obtain full 0 to $2 \pi$ optical phase modulation in a device with a switching angle of $\pm 45^{\circ}$ [23]. However, as noted, fastswitching materials that operate at room temperature tend to show reduced switching angles. In this study, we use a bimesogenic-doped eutectic mixture that exhibits switching angles in the range of $\pm 15^{\circ}$ to $\pm 20^{\circ}$ at room temperature and demonstrate that enhanced optical phase modulation can be obtained by using a multipass geometry.

\section{THEORETICAL CONSIDERATIONS}

\section{A. Phase modulation with a nematic pi cell}

In a layer of nematic LCs with positive dielectric anisotropy, the application of an electric field across the layer causes reorientation of the director from a planar state towards a state with the director oriented perpendicular to the device surfaces. At high voltages, the bulk of the layer is reoriented to the perpendicular state, and small transition regions (or boundary layers) remain near the device surfaces, which are shown in Fig. 1.

In a conventional antiparallel rubbed device that exhibits the well-known Fréedericksz threshold voltage, the director tilt in the boundary layers is in the same sense near the two surfaces. On the other hand, in the so-called pi cell (parallel rubbed surfaces) [27], the tilt is in the opposite sense in the two boundary layers. In the limit of very high voltages, and if the tilt of the director out of the cell surface plane is given by the angle $\theta$ (the tilt angle $\theta$ and coordinate $z$ are defined as in Fig. 1), then these states can be written as

$$
\begin{gathered}
z=0: \theta=0^{\circ}, \\
0<z<d: \theta=90^{\circ}, \\
z=d: \theta=0^{\circ},
\end{gathered}
$$

for the Fréedericksz device and

$$
\begin{gathered}
z=0: \theta=0^{\circ}, \\
0<z<d: \theta=90^{\circ}, \\
z=d: \theta=180^{\circ},
\end{gathered}
$$

for the pi-cell device, where $z$ measures the position through the LC layer. 


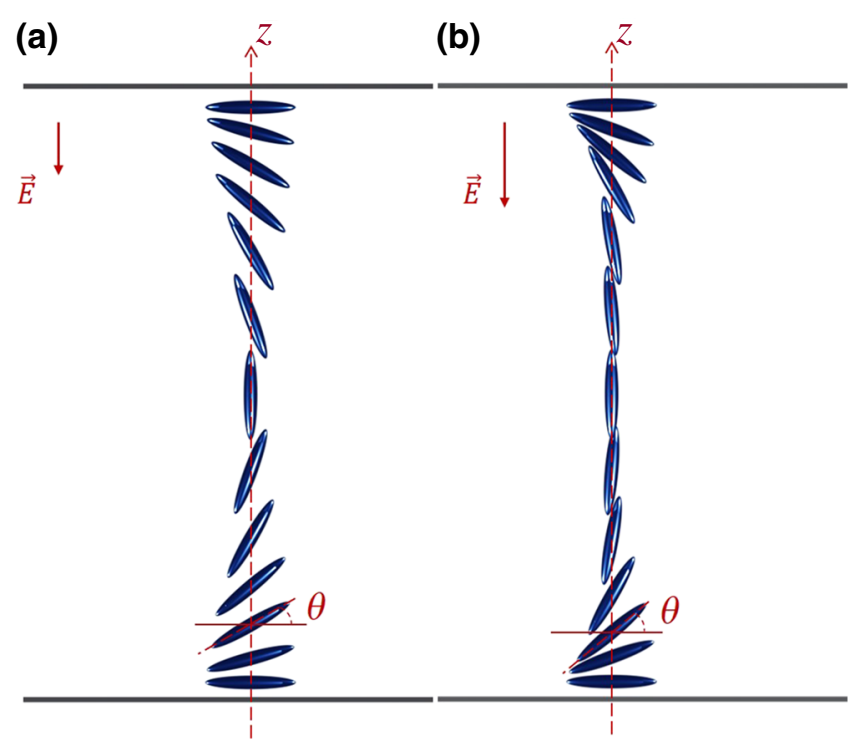

FIG. 1. Orientation of a nematic LC director when subjected to an applied electric field in a pi cell. Orthographic side view of the pi-cell device at (a) low voltage and (b) high voltage. Direction of the electric field in both cases is parallel to the normal of the substrates.

In each case, when the electric field is removed, relaxation of the director from the switched state back towards the planar state begins from the surfaces. If we use a simple one-elastic-constant approximation (i.e., $K_{11}=K_{22}=K_{33}=K$ ) to model the LC behavior, then the initial response of the boundary layers at each surface has an analytic solution in terms of the error function (erf) of the form

$$
\theta(z, t)=\theta_{s}+\left(\theta_{b}-\theta_{s}\right) \operatorname{erf}\left(\frac{z \sqrt{\gamma}}{2 \sqrt{K} \sqrt{t}}\right)
$$

where $z$ is now a coordinate measured from the surface; $\theta_{s}$ and $\theta_{b}$ are the initial surface and bulk director orientations, respectively; and $K$ is the LC elastic constant. This solution is valid at each surface for short timescales provided that the $\operatorname{erf}(\ldots)$ term remains close to unity for $z=d / 2$.

To determine the magnitude of the optical phase change (modulation) available through this boundary-layer behavior, we need to determine the phase, $\phi$, of light propagating through the LC

$$
\phi=\frac{2 \pi}{\lambda} \int_{0}^{d} n_{\mathrm{eff}}(z) d z
$$

where

$$
n_{\mathrm{eff}}(z)=\sqrt{n_{e}^{2} \cos ^{2}(\theta)+n_{o}^{2} \sin ^{2}(\theta)} .
$$

Here, $\lambda$ is the wavelength of light, and $n_{e}$ and $n_{o}$ are the extraordinary and ordinary refractive indices of the
LC, respectively. Further analytic progress is challenging because the integration of the trigonometric function of the error function is nontrivial. However, further progress can be made if (a) we introduce a piecewise linear approximation to the error function of the following form:

$$
\begin{gathered}
\operatorname{erf}(u) \approx \frac{u}{u_{0}}: u<u_{0}, \\
\operatorname{erf}(u) \approx 1: u \geq u_{0},
\end{gathered}
$$

where $u_{0}$ is chosen such that the integral of the approximation to the error function remains correct (leading to $u_{0}=1.12838$ ); and (b) we also expand $n_{\mathrm{eff}}(z)$ for small $\Delta n$.

Assuming that $\theta_{s}=0^{\circ}$ and $\theta_{b}=90^{\circ}, \theta(z, t)$ can now be written as

$$
\begin{gathered}
\theta(z, t) \approx 90^{\circ} \frac{1}{1.12838} \frac{z \sqrt{\gamma}}{2 \sqrt{K} \sqrt{t}} \text { for } \frac{z \sqrt{\gamma}}{2 \sqrt{K} \sqrt{t}}<1.12838 \\
\theta(z, t) \approx 90^{\circ} \text { for } \frac{z \sqrt{\gamma}}{2 \sqrt{K} \sqrt{t}} \geq 1.12838
\end{gathered}
$$

and

$$
n_{\mathrm{eff}}(z) \approx n_{o}\left[1+\frac{A}{2} \cos ^{2}(\theta)-\frac{A^{2}}{8} \cos ^{4}(\theta)+\ldots\right],
$$

where

$$
A=\frac{n_{e}^{2}-n_{o}^{2}}{n_{o}^{2}} .
$$

Substituting these into Eq. (2) allows $\phi$ to be determined as

$$
\phi=\frac{2 \pi}{\lambda}\left[n_{o} d+2 n_{o} z_{\text {crit }}\left(\frac{A}{4}-\frac{3 A^{2}}{64}+\ldots\right)\right],
$$

where

$$
z_{\text {crit }}=1.12838 \frac{2 \sqrt{K} \sqrt{t}}{\sqrt{\gamma}} .
$$

In Eq. (10), the first term in square brackets (the $n_{o} d$ term) represents a fixed-phase term, whereas the other terms represent a change in the phase. Setting the phase change to $\pi / 2$ and using light of wavelength $\lambda=632.8 \mathrm{~nm}$ (He$\mathrm{Ne}$ laser), together with the physical parameters for the nematic LC, E7, allows us to determine a time that represents a response for a $\pi / 2$ phase modulation, which is then estimated to be $t \approx 1 \mathrm{~ms}$. [There is some variation in the published parameters for E7. Based on the elastic 
constants published in Ref. [28], we use a one-constant approximation of $K \approx 15 \mathrm{pN}$. The rotational viscosity published in Ref. [28] is $\gamma \approx 0.08 \mathrm{Pas}$, but values published elsewhere are typically higher. Here, we use a value of $\gamma \approx 0.15 \mathrm{~Pa}$ s. We use $n_{\mathrm{o}}=1.52$ and $n_{e}=1.74$ from Ref. [29]. (All values are quoted at room temperature.)] We note that this response time is independent of the thickness of the LC device provided that its thickness is substantially greater than twice $z_{\text {crit }}$ at this point in time, which is $0.7 \mu \mathrm{m}$ for the above case.

The implication of these findings is that $\pi / 2$ phase modulation is available on a millisecond timescale in the switch-off (relaxation) of nematic layers provided that they are switched on at high voltage. In the experimental Sec. IV A, this behavior is demonstrated using a pi-cell device. By using a multipass configuration, the phase modulation is then enhanced to give a full 0 to $2 \pi$ range.

\section{B. Phase modulation with flexoelectro-optic switching}

In the flexoelectro-optic effect, the applied electric field leads to an in-plane rotation of the effective optic axis of the helical structure by an angle, $\chi$ [the definition is shown in Fig. 2(b)], which to first order is given by [30]

$$
\chi=\frac{e_{1}-e_{3}}{K_{11}+K_{33}} \frac{E}{q}
$$

where $e_{1}$ and $e_{3}$ are the splay and bend flexoelectric coefficients, respectively; $K_{11}$ and $K_{33}$ are the splay and bend elastic constants, respectively; $E$ is the applied electric field; and $q=2 \pi / p$, where $p$ is the pitch of the chiral nematic helix. The orientations of the chiral nematic LCs without and with an applied electric field are shown in Fig. 2.

If the layer of LCs is placed between quarter-wave plates, there is a modulation in the phase of the transmitted light. The magnitude of this phase modulation can be determined using a Jones matrix [31] approach via

$$
E_{\text {out }}=Q\left(-\frac{\pi}{4}\right) F(\chi) Q\left(+\frac{\pi}{4}\right) E_{\text {in }},
$$

where $E_{\text {in }}$ is the incoming light field, $Q(+/-\pi / 4)$ are quarter-wave plates at $\pm 45^{\circ}, F(\chi)$ is the flexoelectro-optic

\section{(a)}

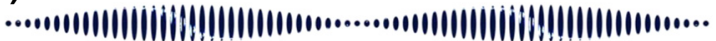

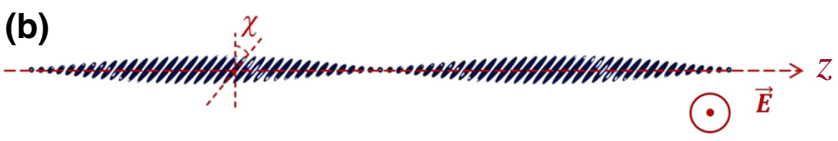

FIG. 2. Definition of the geometrical quantities (tilt angle, $\chi$, and coordinate, $z$ ) for the configuration based on the flexoelectrooptic effect. Device without (a) and with (b) an applied electric field applied perpendicular to the helix axis.
LC layer switched to an angle $\chi$, and $E_{\text {out }}$ is the outgoing light field. Using Eq. (13), it can be demonstrated that the optical phase modulation is twice the switching angle of the effective optic axis in the LC layer. So, if the switching angle is $\pm \chi_{\max }$, then the phase modulation obtained is $\pm 2 \chi_{\max }$. Placing a reflector (mirror) behind this system of components and using the modulator in a reflective mode doubles this further, giving a phase modulation of $\pm 4 \chi_{\max }$. Therefore, with a switching range of $\pm 45^{\circ}$, a phase range of $\pm \pi$ is available (equivalent to a full optical phase range of 0 to $2 \pi$ ).

This principle can be extended further to a "multipass" concept. The single pass, as shown by Eq. (13), leads to a phase modulation of $\pm 2 \chi_{\max }$. The reflection configuration used in previous work [23] is effectively a double-pass arrangement, leading to twice this modulation, i.e., $\pm 4 \chi_{\max }$. Therefore, a three-pass arrangement would lead to $\pm 6 \chi_{\max }$, a four-pass arrangement would lead to $\pm 8 \chi_{\max }$, and so on. In the present study, we demonstrate the enhancement of phase modulation by increasing from a single, to a double, and finally a four-pass arrangement.

\section{EXPERIMENTAL RESULTS AND DISCUSSION}

\section{A. Nematic pi-cell phase modulator}

To begin with, we demonstrate high-speed switching in a nematic pi cell. The pi cell's surfaces are treated so that they can provide a surface alignment that will create the same pretilt direction on both surfaces, so that the flow results in no torque being applied to the LC director in the center of the cell [27]. For this work, the nematic eutectic mixture, E7 (Synthon Ltd.), is capillary filled into a pi cell, which consists of glass substrates that are coated in indium tin oxide (ITO) electrodes and parallel rubbed polyimide alignment layers. The thickness of the device is nominally $5 \mu \mathrm{m}$. Placing such a device between crossed polarizers, with the alignment direction at $45^{\circ}$ to the polarizer axis, the transmission tends to zero at high voltage. When the voltage is removed, the transmission increases. At the point where the transmission reaches $50 \%$, the phase retardation of the device is equivalent to a quarter-wave plate, which is where the phase for light polarized along the alignment direction has changed by $\pi / 2$. This is where the boundary layers have grown to a thickness of $z_{\text {crit }}$, as outlined in Sec. III A.

The behavior described here is shown in Fig. 3, where the wavelength of light used is $\lambda=632.8 \mathrm{~nm}$ (He-Ne laser). The switching voltage waveform is a $5 \mathrm{kHz}$ square wave modulated to give a sequence of $50 \mathrm{~V}_{\mathrm{p} \text {.p. }}$ (drive voltage), $0 \mathrm{~V}$ (relax voltage), and $6 \mathrm{~V}_{\mathrm{p.p}}$. (hold voltage). (The waveform generator is a Tektronix AFG 3022.) First, the $50 \mathrm{~V}_{\text {p.p. }}$ voltage switches the device on before the $0 \mathrm{~V}$ voltage then allows the boundary layers to grow from the surfaces. At the point where the transmission reaches $50 \%$, 


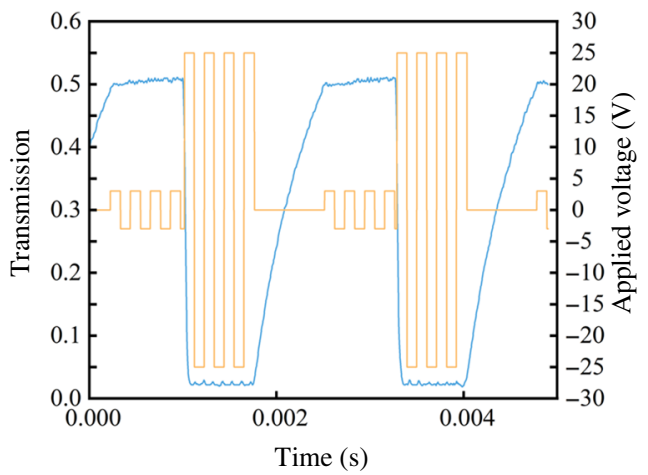

FIG. 3. Transmission as a function of time (blue solid line) for a nematic pi cell between crossed polarizers when the device is driven by the voltage waveform sequence shown by the orange solid line and as described in the main text. Modulation of the transmission by $50 \%$ corresponds to a phase change of $\pi / 2$ in the extraordinary wave.

a holding voltage of $6 \mathrm{~V}_{\text {p.p. }}$ is applied. The sequence is then repeated. Importantly, the time to "relax" to a transmission of $50 \%$ is found to be $0.75 \mathrm{~ms}$. This is actually slightly less than the equivalent time for $\pi / 2$ phase modulation estimated in Sec. III A, but it should be remembered that this is based on a simplified analytic approach.

Next, we place the pi-cell device in a simple MachZehnder interferometer [32]. The experimental arrangement is that shown in Fig. 4(a). The resulting fringes are recorded using a CCD camera, as the device is switched through the sequence described previously; the time of each part of the modulated $5 \mathrm{kHz}$ waveform is now set to be $1 \mathrm{~ms}$ (i.e., $1 \mathrm{~ms}$ at drive voltage, $1 \mathrm{~ms}$ at relax voltage, $1 \mathrm{~ms}$ at hold voltage, repeated). The recorded fringes are then analyzed to track the phase change of the wave front of light passing through the pi-cell device. To demonstrate control of the resulting phase of the transmitted light, the modulation of the $50 \mathrm{~V}_{\text {p.p. }}, 0 \mathrm{~V}$, and $6 \mathrm{~V}_{\text {p.p. }}$. sequence is then adjusted and the phase change is tracked for each case. (Notably, as the modulation of the waveform is decreased, the drive voltage reduces and the relaxation voltage increases, but the overall rms voltage remains sufficient to retain the pi state in the device.) The results are shown in Fig. 5, where the $x$ axis shows the difference between the drive voltage and the relaxation voltage $(\Delta V)$ for different modulation depths (0 to 100\%). In Fig. 5, we can see that a maximum phase modulation of slightly in excess of $90^{\circ}$ is obtained and that this varies smoothly as the modulation is changed. This response is obtained
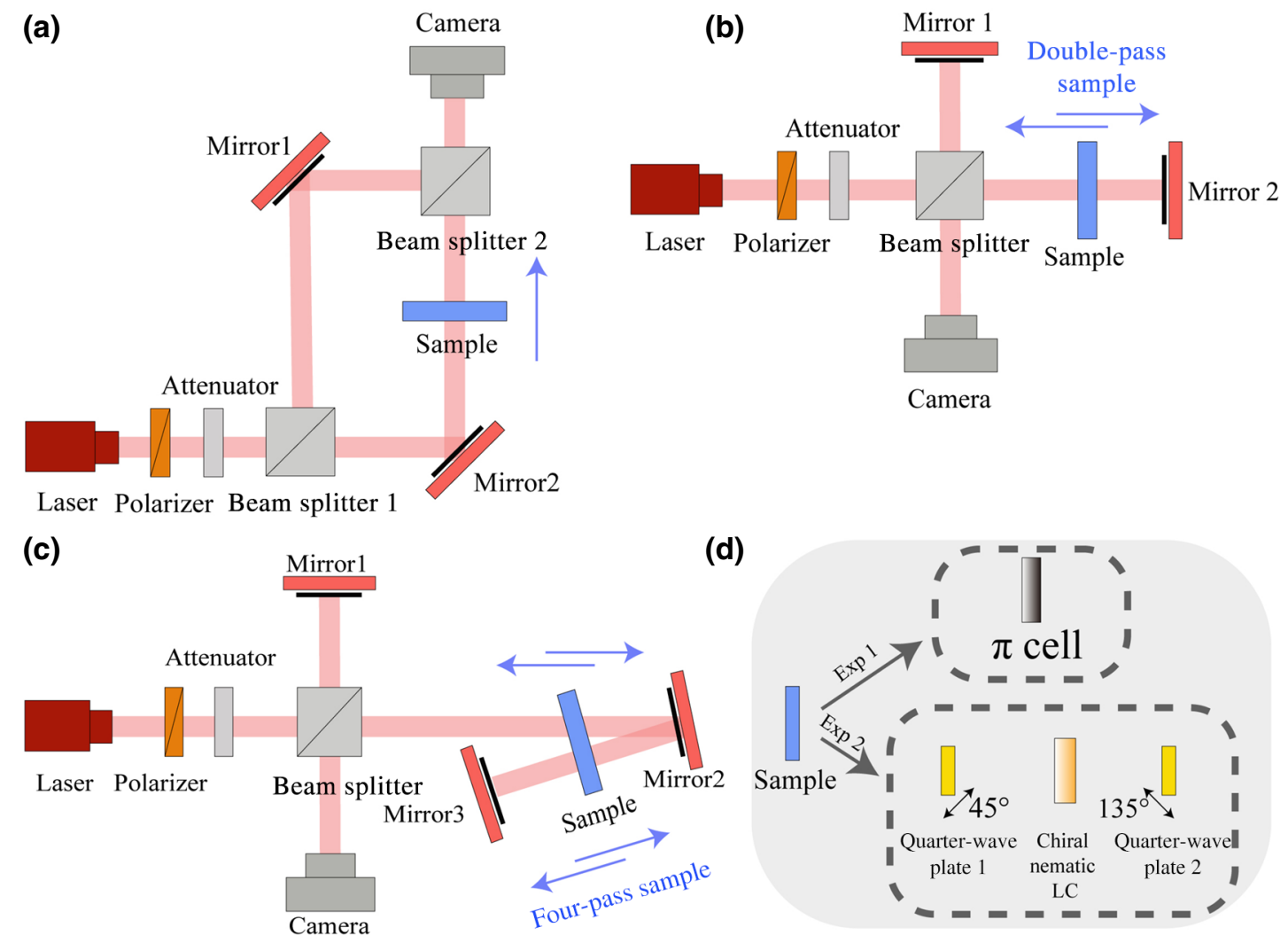

FIG. 4. Schematic diagrams of the experimental arrangements. (a) One-pass setup (Mach-Zehnder interferometer). (b) Two-pass setup (Michelson configuration). (c) Four-pass setup ("folded" Michelson configuration). (d) Two different device geometries are used: a pi cell and a flexoelectro-optic (chiral nematic LC) device between quarter-wave plates. 


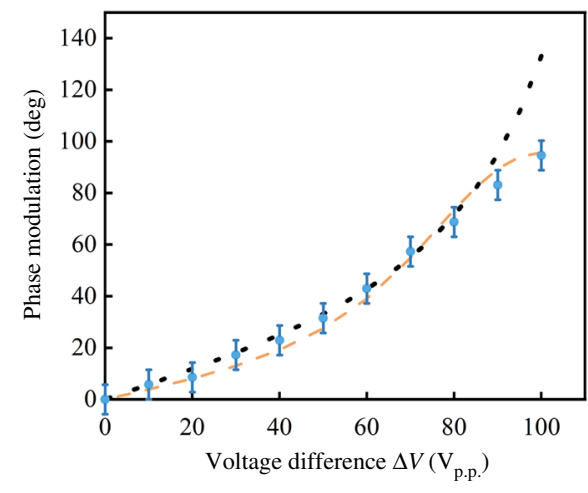

FIG. 5. Phase modulation that is obtained using a pi cell, driven by the waveform sequence, as described in the main text. Data points (blue circles) are from experiments and dashed orange line represents results from the numerical model. Dotted black line shows the results from a simplified analytic model.

in $1 \mathrm{~ms}$. The amplitude (or intensity) modulation in this configuration is minimal because the nematic director is switching in the plane of the incident polarized light. There are small losses due to reflections at the device surfaces and the small amount of optical scattering in the nematic LC, but the overall arrangement is very optically efficient.

Figure 5 also shows the results from modeling the phase behavior. A simple analytic model can be developed based on the ideas introduced in Sec. III A. In this case, a piecewise linear form is again used, but now the boundary-layer thickness, $z_{\text {crit }}$, is determined by minimizing the energy for the driving and holding voltages (this leads to the dotted black line in Fig. 5). Alternatively, rather than using the analytic estimation outlined in Sec. III A, a model can be based on a finite-difference numerical solution to the Euler-Lagrange equations for simple nematic continuum theory. Only the splay and bend elastic constants are relevant in this model (values of $K_{11}=11.1 \mathrm{pN}$ and $K_{33}=17.1 \mathrm{pN}$ are used for E7 [28], which lead to the orange dashed line in Fig. 5). The device thickness is $5 \mu \mathrm{m}$, $\Delta \varepsilon=14.0$, and $\Delta n=0.22$. It can be seen that the simple analytic model is reasonable at lower modulation levels, but diverges substantially at higher modulation levels.
This may be because the dynamic behavior becomes more important in these cases. For the numerical model, it can be seen that, while the overall trend is reasonable, the model falls slightly outside of the error bars associated with the experimental data (measurement of optical phase from the interferometer). It is not entirely clear why this is so, although it may be due to the lack of inclusion of flow in our model, which is generally considered to enhance the switching speeds in pi cells [33]. (Some allowance for this effect is included by reducing the rotational viscosity for the fit, where a value of $\gamma=0.07 \mathrm{~Pa} \mathrm{~s}$ is used, which is very similar to the value quoted in Ref. [28].) In subsequent graphs, we show only the results from the numerical modeling alongside the experimental results.

For the geometry considered here, light is only passing through the device once (i.e., it is a one-pass configuration). Next, we reconfigure the interferometer into a Michelson configuration [34] and place the device in one arm to give a two-pass arrangement. The diagram of the two-pass setup is shown in Fig. 4(b). The light then passes through the device twice, and an enhanced optical phase modulation effect is obtained. The results, for the same driving conditions as those used previously, are shown in Fig. 6(a). We can see from Fig. 6(a) that the behavior follows the same trend as that shown in Fig. 5, but that the optical phase modulation is now twice as much, reaching a maximum in excess of $180^{\circ}(\pi)$.

To increase the modulation depth further, we modify the arrangement of the interferometer to pass light through the device four times; this is done by adding an additional mirror into the signal arm of the Michelson arrangement, as shown in Fig. 4(c). The result for this four-pass configuration is shown in Fig. 6(b). Here, we can see that an optical phase modulation in excess of $360^{\circ}(2 \pi)$ is obtained. Again, we see that the experimental data lie slightly off the line of the model. However, importantly, this configuration demonstrates a full phase modulation of the light (i.e., a phase range of greater than 0 to $2 \pi$ ) with a $1 \mathrm{~ms}$ switching time. Even faster switching times would be available using more optimized materials, such as that used in the work by Huang et al. [14]. (a)

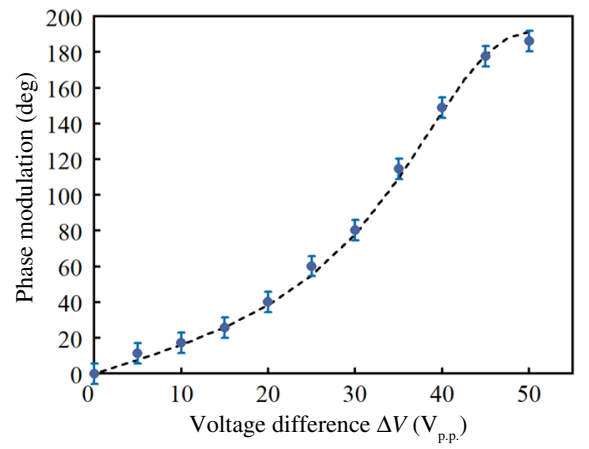

(b)

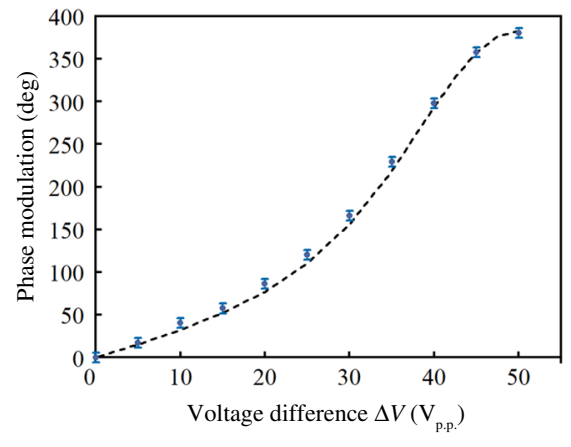

FIG. 6. Phase modulation that is obtained using a pi cell. Points are experimental data and dashed lines are based on the results from the model. (a) Two-pass configuration; (b) four-pass configuration. 


\section{B. Phase modulation with multipass flexoelectro-optic switching}

For the flexoelectro-optic effect, we first investigate the switching behavior of a mixture that consists of a base chiral nematic mixture, comprising E7 + 3 wt \% BDH1281 (Merck) doped with $20 \mathrm{wt} \%$ of the bimesogenic material $4^{\prime}, 4^{\prime}$-(nonane-1,7-diyl)bis[( $\left[1^{\prime}, 1^{\prime \prime}\right.$-biphenyl)$4^{\prime \prime}$-carbonitrile] (CB9CB) $[35,36]$. The chiral nematic mixture, which forms a right-handed helical structure with a pitch of about $520 \mathrm{~nm}$, is capillary filled into a nominally $5-\mu \mathrm{m}$-thick device that consists of glass substrates that are coated with ITO electrodes (to facilitate the application of an electric field along the normal to the device substrates) and antiparallel rubbed polyimide alignment layers. To ensure that the electric field is orthogonal to the helical axis (a necessary requirement to observe flexoelectrooptic switching), a uniform lying helix (ULH) geometry is required. To form the ULH alignment, the device is heated to $50{ }^{\circ} \mathrm{C}$ in the presence of a $500 \mathrm{~Hz}, 25 \mathrm{~V}_{\text {p.p. }}$ square waveform and then cooled to room temperature in the presence of an applied electric field. All measurements are carried out at a temperature of $T=30^{\circ} \mathrm{C}$.

The resulting mixture shows enhanced flexoelectrooptic switching when compared with the base E7 + $3 \mathrm{wt} \%$ BDH1281 mixture, but, in contrast to the neat bimesogenic material, which exhibits a nematic phase at substantially elevated temperatures $\left(>100^{\circ} \mathrm{C}\right)$, this mixture can still be operated at room temperature. The electro-optic properties are investigated by applying a $500 \mathrm{~Hz}$ square-wave voltage waveform using a Tektronix AFG 3022 waveform generator and measuring the switching angle using a combination of a BX51 polarizing microscope, a Tektronix TBS1154 oscilloscope, and high-speed Si photodiode [37]. The results are shown in Fig. 7(a).

In Fig. 7(a), we can see that a peak switching angle of around $35^{\circ}\left( \pm 17.5^{\circ}\right)$ is obtained at a switching voltage of
$50 \mathrm{~V}_{\text {p.p. }}$. However, at this voltage, the helix of the chiral nematic LC begins to unwind and there is some change in the alignment texture; therefore, operation at slightly lower voltages may be preferred. Due to the dielectric anisotropy of the material, and the resulting helix distortion under electric field application, the overall flexoelectrooptic switching behavior is complex. However, the switching angle can be approximated in terms of a cubic series expansion [38]; this is the dashed line in Fig. 7(a). This cubic series can be written in terms of the expression for the flexoelectro-optic tilt angle introduced in Sec. III B, plus a correcting cubic term:

$$
\chi=\frac{e_{1}-e_{3}}{K_{11}+K_{33}} \frac{E}{q}+\beta\left(\frac{e_{1}-e_{3}}{K_{11}+K_{33}} \frac{E}{q}\right)^{3},
$$

where $\beta$ is treated as a fitting parameter and is a complex combination of elastic, dielectric, and flexoelectric properties [38]. From the dashed line in Fig. 7(a), we can determine the ratio $\left(e_{1}-e_{3}\right) /\left(K_{11}+K_{33}\right)$, which is sometimes referred to as the flexoelastic ratio for the material, to be $0.924 \mathrm{~V}^{-1}$ and the value of the fitting parameter $\beta$, which is $\beta=-1.446$; the negative value of $\beta$ indicates that dielectric coupling tends to suppress (reduce) the flexoelectro-optic tilt angle.

We then place this device between quarter-wave plates [Fig. 4(d), Exp2] and insert it into one arm of the MachZehnder interferometer. The device is again driven with a $500 \mathrm{~Hz}$ square wave (so the switching response to either polarity is taking place in $1 \mathrm{~ms}$ ), and the interference fringes are recorded on the CCD camera and tracked to extract the resulting optical phase modulation. The resulting phase modulation is shown in Fig. 7(b). We can see that, as expected, the resulting phase modulation is twice the switching angle of the optic axis in the device because the light passes through the device a single time. However, (a)

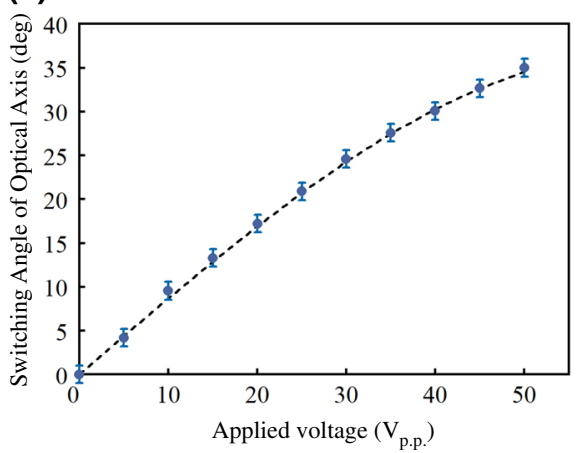

(b)

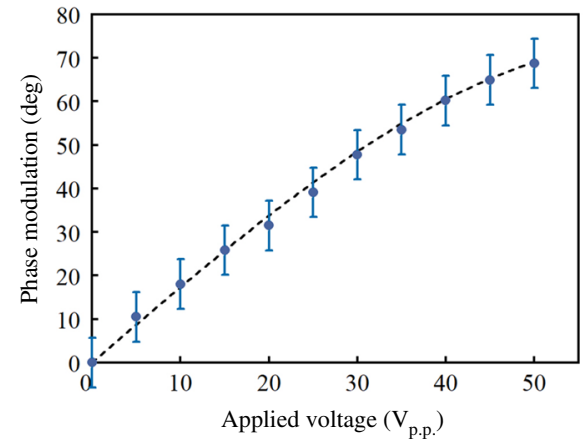

FIG. 7. (a) Flexoelectro-optic switching in the chiral nematic phase of the mixture $\mathrm{E} 7+3 \mathrm{wt} \% \mathrm{BDH} 1281+20 \mathrm{wt} \% \mathrm{CB} 9 \mathrm{CB}$ in a 5 - $\mu$ m-thick device. Applied voltage is a $500 \mathrm{~Hz}$ square wave. (a) Flexoelectro-optic switching angle (as measured on a polarizing microscope) is shown as a function of the applied voltage (peak-to-peak), and dashed line represents a cubic series expansion fit based upon theoretical considerations (see main text). (b) Resulting optical phase modulation in transmission for the same range of flexoelectro-optic switching angles, when the device is placed between a pair of quarter-wave plates. 
this optical phase modulation is still rather small, with a maximum range of $70^{\circ}$ at $50 \mathrm{~V}_{\text {p.p. }}$. There are some losses in this arrangement, due to imperfections in the alignment of the helical axis in the flexoelectro-optic device. These alignment imperfections lead to point defects in the LC structure, which, in turn, result in considerable optical scattering. The demonstration device is therefore somewhat optically inefficient, although it is expected that improvements to the alignment quality would considerably reduce this problem. By choosing the orientation of the device appropriately, there is minimal amplitude (or intensity) modulation in this configuration. This is estimated to be less than $10 \%$ in amplitude (less than $1 \mathrm{~dB}$ ).

The phase modulation depth can be enhanced if we instead place the device (still between quarter-wave plates) in one arm of the Michelson interferometer. In this case, light now passes through the device twice. This is the configuration that we have previously presented using a different mixture that exhibits large switching angles, but at very high temperatures $\left(108^{\circ} \mathrm{C}\right)$ [23]. The result for the present device is shown in Fig. 8(a). It is immediately noticeable in Fig. 8(a) that the phase modulation has a different curvature from that shown in Fig. 7 for the switching angle and single-pass phase modulation behavior, with the curvature being reversed at lower voltages. Figure 8(b), which presents the result when light passes through the device four times, shows similar qualitative behavior to the two-pass case. This effect happens because of the change in retardation of the LC layer under an applied voltage. The material used here has a significant dielectric anisotropy (as noted earlier, for E7 only at room temperature $\Delta \varepsilon=14$ ), and therefore, under the application of the electric field there is a tendency for the helix of the chiral nematic LC to distort. In the configuration used here, with the electric field direction and light propagation direction perpendicular to the helical axis, this leads to a reduction in the effective optical anisotropy as the electric field amplitude is increased. To first order, this reduction is quadratic in the applied electric field [38].

When the flexoelectro-optic LC device is placed between a pair of quarter-wave plates and the phase shift is measured using polarized light [the configuration for the one-pass transmission measurement results shown in Fig. 7(b)], then the phase modulation follows the tilt angle of the optical axis, as can be seen by comparing Figs. 7(a) and 7(b). However, when a mirror is placed behind the second quarter-wave plate, the behavior changes. The configuration experienced by the light path is now quarter-wave plate, LC device (first pass), quarter-wave plate, mirror, quarter-wave plate, LC device (second pass), and quarterwave plate. Now, changes in the effective birefringence of the LC layer under the application of an electric field distorts the optical phase change as a function of tilt angle behavior, making it nonlinear and resulting in the curves seen in Fig. 8. The shape of these curves would be qualitatively similar to those shown in Fig. 7 if a polarizer were placed between the second quarter-wave plate and the mirror.

In Fig. 7(a), the switching angle of the flexoelectro-optic LC device for a voltage of $45 \mathrm{~V}_{\text {p.p. }}$ at $500 \mathrm{~Hz}$ is over a range of $32.5^{\circ}$ (i.e., $\pm 16.25^{\circ}$ ). The resulting optical phase change available in a one-pass configuration is then twice this value, which is $65^{\circ}$ for $45 \mathrm{~V}_{\text {p.p. }}$, as shown in Fig. 7(b). Although the shapes of the curves are different, we can see that, in the two-pass case, the maximum optical phase modulation is again doubled [to a range of $130^{\circ}$ for $45 \mathrm{~V}_{\text {p.p. }}$. in Fig. 8(a)]. In the four-pass case, it is further doubled to a maximum range of $260^{\circ}$ for $45 \mathrm{~V}_{\text {p.p. }}$, as seen in Fig. $8(\mathrm{~b})$. At this point, the optical phase modulation is 8 times the flexoelectro-optic switching angle range, which means that full 0 to $2 \pi$ optical phase modulation would be available (a)

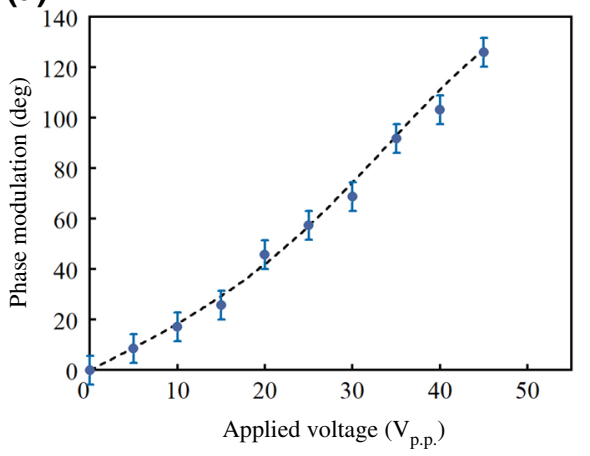

(b)

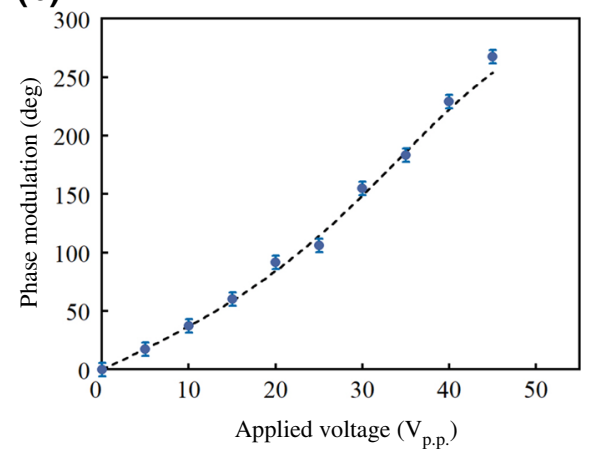

FIG. 8. Phase modulation as a function of applied voltage (peak-to-peak) for the flexoelectro-optic device, consisting of the chiral nematic mixture $\mathrm{E} 7+3 \mathrm{wt} \% \mathrm{BDH} 1281+20 \mathrm{wt} \% \mathrm{CB} 9 \mathrm{CB}$, for multipass geometries: (a) two-pass and (b) four-pass configurations. Thickness of the device is nominally $5 \mu \mathrm{m}$. Data points represent results from the experiments, and results from the model(s) are black dashed lines. Precise form of the behavior depends on the orientation of the device, which, in this case, is chosen to minimize the amplitude (or intensity) modulation to keep it below $10 \%$. 
in this arrangement using a flexoelectro-optic device with a switching angle of $45^{\circ}\left( \pm 22.5^{\circ}\right)$.

\section{CONCLUSIONS}

In summary, we demonstrate two different configurations that allow substantial optical phase modulation on a millisecond timescale at room temperature. Using a pi cell driven at high voltages in a multipass optical arrangement allows a boundary-layer high-speed switching effect to be exploited, giving full 0 to $2 \pi$ optical phase modulation in $1 \mathrm{~ms}$. It should be noted that this device uses the well-characterized nematic LC, E7, and therefore, substantially faster switching times could be obtained by using an optimized mixture (e.g., one with lower rotational viscosity, higher optical birefringence, larger elastic constants). This would therefore pave the way to full 0 to $2 \pi$ optical phase modulation on submillisecond timescales.

Using the flexoelectro-optic effect in chiral nematic LC devices, we show enhanced multipass optical phase modulation. Modulation of $260^{\circ}$ is achieved on a $1 \mathrm{~ms}$ timescale using a material that shows a switching angle of just over $32^{\circ}$ at $45 \mathrm{~V}_{\text {p.p. }}$. Full 0 to $2 \pi$ optical phase modulation can be achieved either using a slightly more optimized mixture (to give a switching angle of $\pm 22.5^{\circ}$ ) or by using a six-pass optical arrangement. For example, a material developed by Varanytsia and Chien based on the nematic host MLC-2048 (Merck) doped with a bimesogenic compound, $\mathrm{CB} 7 \mathrm{CB}$, shows room-temperature switching angles of up to $\pm 40^{\circ}$ [22], although this is at somewhat higher applied electric field amplitudes.

A key application of the ideas proposed here is in the development of spatial light modulator technology with submillisecond switching over a full phase range. To implement the multipass configurations using such technology, it would be necessary to ensure that each modulating pixel is reimaged onto the same modulating pixel in subsequent passes. For two-pass operation, this can be achieved by integrating a mirror into the back plane of the device. For four-pass (and higher) arrangements, there are two possible approaches. For example, Peng et al. have demonstrated a very interesting integrated multipass configuration when optimizing the phase modulation available using blue-phase LC technology [39]. This solution is very elegant, but the integrated mirrors do substantially reduce the useable mark-space ratio of the pixels in a device. An alternative approach to this would necessitate an imaging system, such as a folded four- $f$ system. In this arrangement, light is incident on a SLM with an integrated mirror back plane. The reflected light is then passed through a lens, which is placed its focal length from the spatial light modulator, and onto a mirror, which is placed at the focal length of the lens behind the lens. The light is hence reflected and precisely imaged back through the lens onto the same pixel in the SLM through which it first passed. The light therefore passes four times through each individual pixel. Further higher-pass configurations can be envisaged, although the imaging arrangements would not be convenient to implement.

A further concern for the practical technological implementation of the ideas presented here are the drive voltages used. These are up to $50 \mathrm{~V}_{\text {p.p. }}( \pm 25 \mathrm{~V})$, which are somewhat higher than those normally used in spatial light modulators with integrated silicon back planes. However, it is anticipated that these drive voltages could be reduced through material choice and/or optimization. In the pi-cell demonstration presented here, the work is undertaken with standard nematic material, E7. Choosing (or potentially developing) a material with higher dielectric anisotropy and high optical birefringence would allow the drive voltages to be substantially reduced. The flexoelectro-optic phase modulator is constructed using an in-house developed mixture. Again, optimization of the material to enhance its flexoelectric coefficient and increase its birefringence would allow the use of thinner layers at reduced voltages.

In future work, we aim to develop the concepts introduced here to demonstrate multipixel phase modulators. These have exciting applications in areas such as optical beam steering, programmable diffractive elements, and micromanipulation.

\section{ACKNOWLEDGMENTS}

This research is supported by the Engineering and Physical Sciences Research Council (UK) (Grants No. EP/ M017923/1 and No. EP/M015726/1), and the European Space Agency (Contract No. 4000125232/18/NL/AR/zk).

[1] S. Mias and H. Camon, A review of active optical devices: II. Phase modulation, J. Micromech. Microeng. 18, 083002 (2008).

[2] D. G. Grier, A revolution in optical manipulation, Nature 424, 810 (2003).

[3] C. Maurer, A. Jesacher, S. Bernet, and M. RitschMarte, What spatial light modulators can do for optical microscopy, Laser Photonics Rev. 5, 81 (2011).

[4] R. Lynge Eriksen, V. Ricardo Daria, J. Glückstad, R. Eriksen, V. Daria, and J. Gluckstad, Fully dynamic multiplebeam optical tweezers, Opt. Express 10, 597 (2002).

[5] D. J. McKnight, K. M. Johnson, and R. A. Serati, $256 \times$ 256 liquid-crystal-on-silicon spatial light modulator, Appl. Opt. 33, 2775 (1994).

[6] J. A. Davis, D. E. McNamara, D. M. Cottrell, and T. Sonehara, Two-dimensional polarization encoding with a phaseonly liquid-crystal spatial light modulator, Appl. Opt. 39, 1549 (2000).

[7] N. Chattrapiban, E. A. Rogers, D. Cofield, W. T. Hill, III and R. Roy, Generation of nondiffracting Bessel beams by use of a spatial light modulator, Opt. Lett. 28, 2183 (2003). 
[8] S. E. Broomfield, M. A. A. Neil, E. G. S. Paige, and G. G. Yang, Programmable binary phase-only optical device based on ferroelectric liquid crystal SLM, Electron. Lett. 28, 26 (1992).

[9] L. Vicari, Optical Applications of Liquid Crystals (CRC Press, Boca Raton, Florida, USA, 2016), pp. 41-57.

[10] S. P. Kotova and A. M. Mayorova, E. P. Pozhidaev and S. A. Samagin, Simulation of spatial phase light modulators based on the ferroelectric liquid-crystals, J. Phys.: Conf. Ser. 1096, 012017 (2018).

[11] Z. Feng and K. Ishikawa, Phase modulator mode based on the pre-transitional effect of antiferroelectric liquid crystals, Opt. Lett. 43, 251 (2018).

[12] U. Efron, S. T. Wu, and T. D. Bates, Nematic liquid crystals for spatial light modulators: Recent studies, J. Opt. Soc. Am. B 3, 247 (1986).

[13] J. Albero, P. García-Martínez, J. Luis Martínez, and I. Moreno, Second order diffractive optical elements in a spatial light modulator with large phase dynamic range, Opt. Lasers Eng. 51, 111 (2013).

[14] Y. Huang, Z. He, and S.-T. Wu, Fast-response liquid crystal phase modulators for augmented reality displays, Opt. Express 25, 32757 (2017).

[15] E. Hallstig, J. Stigwall, M. Lindgren, and L. Sjoqvist, Laser beam steering and tracking using a liquid crystal spatial light modulator, Proc. SPIE 5087, 13 (2003).

[16] A. Jesacher and M. Booth, Parallel direct laser writing in three dimensions with spatially dependent aberration correction, Opt. Express 18, 21090 (2010).

[17] Y. Lee, D. Franklin, F. Gou, G. Liu, F. Peng, D. Chanda, and S.-T. Wu, Two-photon polymerization enabled multi-layer liquid crystal phase modulator, Sci. Rep. 7, 16260 (2017).

[18] Z. He, G. Tan, D. Chanda, and S.-T. Wu, Novel liquid crystal photonic devices enabled by two-photon polymerization, Opt. Express 27, 11472 (2019).

[19] Z. He, Y.-H. Lee, F. Gou, D. Franklin, D. Chanda, and S.$\mathrm{T}$. Wu, Polarization-independent phase modulators enabled by two-photon polymerization, Opt. Express 25, 33688 (2017).

[20] Z. He, F. Gou, R. Chen, K. Yin, T. Zhan, and S.-T. Wu, Liquid crystal beam steering devices: Principles, recent advances, and future developments, Crystals 9, 292 (2019).

[21] J. S. Patel and R. B. Meyer, Flexoelectric Electro-Optics of a Cholesteric Liquid Crystal, Phys. Rev. Lett. 58, 1538 (1987).

[22] A. Varanytsia and L. C. Chien, Giant flexoelectro-optic effect with liquid crystal dimer CB7CB, Sci. Rep. 7, 41333 (2017).

[23] J. A. J. Fells, X. Wang, S. J. Elston, C. Welch, G. H. Mehl, M. J. Booth, and S. M. Morris, Flexoelectro-optic liquid crystal analog phase-only modulator with a $2 \pi$ range and $1 \mathrm{kHz}$ switching, Opt. Lett. 43, 4362 (2018).
[24] S-T Wu and C-S Wu, High-speed liquid-crystal modulators using transient nematic effect, J. Appl. Phys. 65, 527 (1989).

[25] R. Chen, Y. Huang, J. Li, M. Hu, J. Li, X. Chen, P. Chen, S.-T. Wu, and Z. An, High-frame-rate liquid crystal phase modulator for augmented reality displays, Liq. Cryst. 46, 309 (2019).

[26] G. D. Love, A. K. Kirby, and R. A. Ramsey, Submillisecond, high stroke phase modulation using polymer network liquid crystals, Opt. Express 18, 7384 (2010).

[27] P. J. Bos and K. Rickey Koehler/beran, The pi-cell: A fast liquid-crystal optical-switching device, Mol. Cryst. Liq. Cryst. 113, 329 (1984).

[28] W. Ye, Z. Li, R. Yuan, P. Zhang, T. Sun, M. Cai, X. Wang, J. Zhu, Y. Sun, and H. Xing, Accurate measurement of the twist elastic constant of liquid crystal by using capacitance method, Liq. Cryst. 46, 349 (2019).

[29] P. C.-P. Chao, Y.-Y. Kao, and C.-J. Hsu, A new negative liquid crystal lens with multiple ring electrodes in unequal widths, IEEE Photonics J. 4, 250 (2012).

[30] B. I. Outram and S. J. Elston, Frequency-dependent dielectric contribution of flexoelectricity allowing control of state switching in helicoidal liquid crystals, Phys. Rev. E 88, 012506 (2013).

[31] P. Yeh and C. Gu, Optics of Liquid Crystal Displays (John Wiley \& Sons, Inc., New York, USA, 2009), Chap 4, pp. 173-277.

[32] C. S. Caldwell, J. R. Hall, and A. L. Babb, Mach-Zehnder interferometer for diffusion measurements in volatile liquid systems, Rev. Sci. Instrum. 28, 816 (1957).

[33] H. G. Walton and M. J. Towler, On the response speed of pi-cells, Liq. Cryst. 27, 1329 (2000).

[34] C. Sun, Multiplexing of fiber-optic acoustic sensors in a Michelson interferometer configuration, Opt. Lett. 28, 1001 (2003).

[35] C. Bacchiocchi, M.-G. Tamba, G. H. Mehl, A. Arcioni, I. Miglioli, and C. Zannoni, EPR study of the polydomain structure of the twist-bend nematic phase of CB9CB in the bulk, Liq. Cryst. 45, 2109 (2018).

[36] V. P. Panov, R. Balachandran, M. Nagaraj, J. K. Vij, M. G. Tamba, A. Kohlmeier, and G. H. Mehl, Microsecond linear optical response in the unusual nematic phase of achiral bimesogens, Appl. Phys. Lett. 99, 261903 (2011).

[37] F. Castles, S. C. Green, D. J. Gardiner, S. M. Morris, and H. J. Coles, Flexoelectric coefficient measurements in the nematic liquid crystal phase of 5CB, AIP Adv. 2, 022137 (2012).

[38] D. R. Corbett and S. J. Elston, Modeling the helical flexoelectro-optic effect, Phys. Rev. E 84, 041706 (2011).

[39] F. Peng, Y.-H. Lee, Z. Luo, and S.-T. Wu, Low voltage blue phase liquid crystal for spatial light modulators, Opt. Lett. 40, 5097 (2015). 